\title{
Saikosaponin D inhibits proliferation of human osteosarcoma cells via the p53 signaling pathway
}

\author{
LIN ZHAO ${ }^{1}$, JING LI ${ }^{2}$, ZHI-BO SUN ${ }^{1}$, CHEN SUN $^{1}$, ZHI-HONG YU $^{1}$ and XIAO GUO ${ }^{1}$ \\ ${ }^{1}$ Department of Orthopaedics, Renmin Hospital, Hubei University of Medicine, Shiyan, \\ Hubei 442000; ${ }^{2}$ Department of Dermatology, Chongqing First People's Hospital and \\ Chongqing Traditional Chinese Medicine Hospital, Chongqing 400011, P.R. China
}

Received April 23, 2018; Accepted September 28, 2018

DOI: $10.3892 /$ etm.2018.6969

\begin{abstract}
Saikosaponin D (SSd), the major monomeric terpenoid extracted from Radix bupleuri, a traditional Chinese medicinal herb, exerts various pharmacological properties, including antitumor, anti-inflammatory and antiviral. The present study aimed to investigate the role of SSd in human osteosarcoma (OS) cell growth. In the investigation MTS and EdU assays were applied and flow cytometric analyses of cell cycle and apoptosis were performed. Western blotting and reverse transcription-quantitative polymerase chain reaction analyses were used to explore the underlying mechanisms of SSd on cell cycle transition and p53 signaling. Here, it was demonstrated that $\mathrm{SSd}$ administration at $80 \mu \mathrm{mol} / 1$ significantly inhibited $143 \mathrm{~B}$ and MG-63 proliferation. Furthermore, SSd significantly increased the percentage of 143B and $M G-63$ cells in $G_{0}-G_{1}$ phase and the number of apoptosis cells compared with the control group. Data further demonstrated that SSd treatment upregulated mRNA and protein levels of tumor protein 53 (p53) and its downstream targets, including p21, p27, B-cell lymphoma-2-like protein 4 and cleaved caspase-3, and downregulated mRNA and protein levels of cyclinD1. The results suggested that SSd was a functional tumor suppressor and inhibited OS proliferation via activation of the p53 signaling pathway and may be used in the treatment of osteosarcoma in future.
\end{abstract}

\section{Introduction}

Osteosarcoma (OS) is characterized by the direct formation of immature bone or osteoid tissue by malignant cells that occurs in children and adults (1). According to an estimate by the National Cancer Institute in 2017, OS accounted for $0.2 \%(3,260)$

Correspondence to: Professor Xiao Guo, Department of Orthopaedics, Renmin Hospital, Hubei University of Medicine, 39 Shiyan Chao-yang Road, Shiyan, Hubei 442000, P.R. China E-mail: 2807108146@qq.com

Key words: saikosaponin D, osteosarcoma, tumor suppressor, proliferation, tumor protein 53 of all new cancer cases and $0.3 \%(1,550)$ of cancer worldwide mortality (2). Despite advance in diagnosis and treatment of OS, including magnetic resonance imaging, neoadjuvant chemotherapy and surgery, the overall survival rate remains poor $(<30 \%)$ due to the invasiveness and distant metastasis largely affecting the lung $(3,4)$. Therefore, novel effective and reliable methods are required in the treatment of OS.

Saikosaponin D (SSd) is one of the main bioactive ingredients extracted and purified from Radix bupleuri L, which is prescribed in traditional Chinese medicine for inflammatory, infectious and vascular diseases or as a hepatoprotective, antibacterial or antiviral $(3,4)$. Previous research reported that SSd exhibits significant antitumor activities in cancer cells, including breast, lung, ovarian and pancreatic cancer and certain types of OS (5-12). SSd induces apoptosis in DU145 pancreatic cancer cells via intrinsic apoptotic pathways and sensitizes cancer cells to cisplatin through ROS-mediated apoptosis, which in combination with further saikosaponins may be an effective therapeutic strategy. Furthermore, SSd inhibited hepatocellular carcinoma (HCC) development and downregulated syndecan-2, matrix metalloproteinase (MMP)-2, MMP-13 and tissue inhibitor of metalloproteinase-2 expression in HCC liver tissue, suggesting SSd is a potential candidate in antitumor treatment in OS.

In previous studies, the potential antitumor mechanism of SSd influencing apoptosis and autophagic cell death was demonstrated $(7-9,13)$. However, biological functions and associated molecular mechanisms of SSd in OS have not yet been elucidated. In the present research, tumor-suppressive functions and mechanisms of SSd in OS were evaluated in order to test the potential of using SSd as an antitumor drug in OS.

\section{Materials and methods}

Cell culture and chemicals. Human OS cells lines (143B and MG-63) were purchased from the American type culture collection (Manassas, VA, USA). Cell lines were cultured in RPMI-1640 containing 10\% fetal bovine serum (both Gibco; Thermo Fisher Scientific, Inc., Waltham, MA, USA) at $37^{\circ} \mathrm{C}$ in a $5 \% \mathrm{CO}_{2}$ humidified atmosphere, as previously described (14). SSd (purity, >95\%; authenticated by Shanghai Academy of Life Sciences, Shanghai, China) was mixed with RPMI-1640 medium and stored at room temperature. 
Nucleic acid extraction. $143 \mathrm{~B}$ and $\mathrm{MG}-63$ cells (1x10 ${ }^{4}$ cells/well) were cultured in 6-well plates in RPMI-1640 medium supplemented with $10 \%$ FBS for $24 \mathrm{~h}$ at room temperature. The medium was replaced with RPMI-1640 supplemented with $10 \%$ FBS containing $80 \mu \mathrm{mol} / 1 \mathrm{SSd}$ and cells were cultured for $48 \mathrm{~h}$ at $37^{\circ} \mathrm{C}$ prior to harvesting. RNA was extracted as reported previously (15). Total RNA was isolated from the cells using TRIzol reagent (Thermo Fisher Scientific, Inc.). Isolated RNA samples were quantified using spectrophotometry and stored at $-80^{\circ} \mathrm{C}$ until further use. RNA concentrations were detected by a NanoDrop 2000 spectrophotometer (Thermo Fisher Scientific, Inc.) and $2 \mu \mathrm{l}$ samples were separated using gel electrophoresis on a $5 \%$ agarose gel to examine the purity of the RNA as reported previously $(15,16)$.

MTS assay. Cell viability was assessed by MTS assay (Beyotime Institute of Biotechnology, Haimen, China). Cells (143B and MG-63) were cultured in 96-well plates (2,000 cells/well) RMPI-1640 supplemented with $10 \%$ FBS for $24 \mathrm{~h}$ at room temperature $(14,17)$. Cells were incubated with RMPI-1640 plus $10 \%$ FBS and varying concentrations of $\operatorname{SSd}(20,40,60$ and $80 \mu \mathrm{mol} / \mathrm{l})$ for 24,48 and $72 \mathrm{~h}$ at room temperature. Following, $20 \mu \mathrm{l}$ MTS solution was added to each well and samples were incubated at room temperature for $3 \mathrm{~h}$ prior to absorbance measurements at $450 \mathrm{~nm}$ using a microplate reader. Each experiment was repeated three times.

EdU assay. EdU assays were performed using the Click-iT EdU Imaging kit (Promega Corporation, Madison, WI, USA) and an apollo fluorescent dye (Guangzhou RiboBio Co., Ltd., Guangzhou, China) in accordance with the manufacturer's instructions. 143B and MG-63 cells $\left(3 \times 10^{3}\right.$ cells/well) were incubated in RMPI-1640 medium plus $10 \%$ FBS with $80 \mu \mathrm{mol} / 1 \mathrm{SSd}$ for 24 and $48 \mathrm{~h}$ at room temperature. Cells were exposed to $100 \mu \mathrm{l} \mathrm{EdU}$ at $37^{\circ} \mathrm{C}$ for $3 \mathrm{~h}$ and fixed at room temperature for $20 \mathrm{~min}$ with $4 \%$ paraformaldehyde. The number of EdU-positive and total cells was counted using a fluorescence microscope (magnification in blue light, x20) with four non-overlapping fields per coverslip. Each experiment was repeated three times.

Flow cytometry analysis of cell cycle and apoptosis. Cell cycle and apoptosis were determined by flow cytometry as previously described (18). For cell cycle assays, cells were plated in six-well plates at $1 \times 10^{5}$ cells/well and cultured overnight at room temperature. RMPI-1640 medium plus $10 \% \mathrm{FBS}$ with $80 \mu \mathrm{mol} / 1 \mathrm{SSd}$ were added to the plates for $48 \mathrm{~h}$ and cells were collected, digested and centrifuged ( $800 \mathrm{x} \mathrm{g}$ at room temperature for $10 \mathrm{~min}$ ). Following, cells were incubated with ice-cold $70 \%$ ethanol at room temperature for $24 \mathrm{~h}$ and treated with propidium iodide (PI) for $30 \mathrm{~min}$ at $4{ }^{\circ} \mathrm{C}$ in the dark. Annexin V-fluorescein isothiocyanate (FITC)/PI staining was performed for apoptosis analysis following the manufacturer's guidelines (BD Pharmingen; BD Biosciences, Franklin Lakes, NJ, USA) for $30 \mathrm{~min}$ at $4^{\circ} \mathrm{C}$ in the dark. Samples were analyzed using a flow cytometer. Data were analyzed using CellQuest 16.0 software (BD Biosciences). Each experiment was repeated three times. Apoptosis assays were performed using the described procedure and staining was based on FITC-Annexin V and PI using the FITC-Annexin V Apoptosis Detection kit (BD Biosciences) according to manufacturer's instructions. Experiments were performed in triplicate.

Reverse transcription-quantitative polymerase chain reaction (RT-qPCR) analysis. RNA samples extracted from control and SSd-treated cells were reverse transcribed using a Go-Taq DNA polymerase kit (Promega Corporation) as reported previously $(18,19)$. RT was performed with the following protocol: $37^{\circ} \mathrm{C}$ for $15 \mathrm{~min}$ and $85^{\circ} \mathrm{C}$ for $5 \mathrm{sec}$. $\beta$-actin functioned as the internal control. RT-qPCR reactions were performed using the HT7500 system (Applied Biosystems; Thermo Fisher Scientific, Inc.) and qPCR was performed using the SYBR-Green PCR Master mix (Thermo Fisher Scientific, Inc.). The PCR program began with an initial denaturation at $95^{\circ} \mathrm{C}$ for $2 \mathrm{~min}$, followed by 40 amplification reaction cycles $\left(95^{\circ} \mathrm{C}\right.$ for $30 \mathrm{sec}, 55^{\circ} \mathrm{C}$ for $30 \mathrm{sec}$ and $72^{\circ} \mathrm{C}$ for $30 \mathrm{sec}$ ), with a final extension at $72^{\circ} \mathrm{C}$ for $3 \mathrm{~min}$. Primer sequences are reported in Table I. Each sample was tested in triplicate and relative amounts of mRNA were determined using the $2^{-\Delta \Delta C t}$ method (20).

Western blot analysis. Proteins from cells cultured in RMPI-1640 medium plus 10\% FBS with $80 \mu \mathrm{mol} / 1 \mathrm{SSd}$ were collected using RIPA buffer containing fresh protease and phosphatase inhibitor cocktails (all EMD Millipore, Billerica, MA, USA). To determine the concentration of proteins, a BCA protein kit (Thermo Fisher Scientific, Inc.) was used. Extracted proteins (50 $\mu \mathrm{g} /$ lane) were separated as previously described (21). Proteins was separated by SDS-PAGE on a $10 \%$ gel and transferred to polyvinylidene difluoride membranes (EMD Millipore). Aliquoted proteins were separated with $12 \%$ gels using SDS-PAGE and then transferred to polyvinylidene fluoride membranes. After blocking with $5 \%$ nonfat milk at room temperature for $1 \mathrm{~h}$, the membranes were incubated with primary antibodies at $4^{\circ} \mathrm{C}$ overnight. The following antibodies were included: Tumor protein 53 (p53; cat. no. ab131442); p21 (cat. no. ab188224); p27 (cat. no. ab190851); cyclinD1 (cat. no. ab137875); B-cell lymphoma (Bcl)-2-like protein 4 (Bax; cat. no. ab182733); cleaved caspase-3 (cat. no. ab198447; all 1:1,000); and $\beta$-actin (cat. no. ab8227; 1:2,000; all Abcam, Cambridge, UK) as a control. Membranes were then incubated with horseradish peroxidase-conjugated secondary goat anti-rabbit antibodies (cat. no. sc-2004; 1:5,000; Santa Cruz Biotechnology, Inc., Dallas, TX, USA) at room temperature for $1 \mathrm{~h}$. The bound antibodies were detected using enhanced chemiluminescence kit (EMD Millipore) and protein blots were visualized with the Las4000 Imaging system (GE Healthcare Life Sciences, Little Chalfont, UK).

Statistical analysis. SPSS 17.0 (SPSS, Inc. Chicago, IL, USA) was used for statistical analyses. All data are expressed as means \pm standard deviation. Differences between two samples were assessed by two-tailed Student's t-test and one-way analysis of variance followed by the Bonferroni post hoc test to compare between multiple groups. $\mathrm{P}<0.05$ was considered to indicate a statistically significant difference. 
Table I. Primers used in reverse transcription-quantitative polymerase chain reaction analysis.

\begin{tabular}{llc}
\hline Gene & \multicolumn{1}{c}{ Sequence (5'-3') } & $\begin{array}{c}\text { Fragment } \\
\text { length } \\
\text { (bp) }\end{array}$ \\
\hline p53-F & TACACTGCCCAGGAGCCAGA & 103 \\
p53-R & TGGCACCAGTGTCCGGATTA & \\
p21-F & TGGAGACTCTCAGGGTCGAAA & 65 \\
p21-R & GGCGTTTGGAGTGGTAGAAATC & \\
p27-F & CGTGCGAGTGTCTAACGG & 206 \\
p27-R & CGGATCAGTCTTTGGGTC & \\
CyclinD1-F & CTAGCAAGCTGCCGAACC & 90 \\
CyclinD1-R & TCCGAGCACAGGATGACC & \\
Bax-F & TCCACCAAGAAGCTGACGAG & 275 \\
Bax-R & GTCCAGCCCATGATGGTTCT & \\
Caspase-3-F & ATGGAGAACAATAAAACCCT & 50 \\
Caspase-3-R & CTAGTGATAAAAGTAGAGTTC & \\
$\beta$-actin-F & TCCTGTGGCATCCACGAAACT & 315 \\
$\beta$-actin-R & GAAGCATTTGCGGTGGACGAT & \\
\hline
\end{tabular}

P, tumor protein; Bax, B-cell lymphoma-2-like protein 4; F, forward; $\mathrm{R}$, reverse.

\section{Results}

SSd inhibits cell proliferation. 143B and MG-63 cell viability and proliferation were tested by MTS and EdU assays. MTS assays assessed effects of varying concentration of SSd $(20,40,60$ and $80 \mu \mathrm{mol} / \mathrm{l})$ on cell viability and results demonstrated that SSd had no significant effects at 20-60 $\mu \mathrm{mol} / 1$ when compared with the control group ( $\mathrm{P}>0.05$; Fig. 1A). At $80 \mu \mathrm{mol} / \mathrm{l} \mathrm{SSd}$ significantly inhibited $143 \mathrm{~B}$ and MG-63 cell viability following 24 and $48 \mathrm{~h}$ treatment compared with the control $(\mathrm{P}<0.001$; Fig. 1A). EdU incorporation assays further suggested that SSd significantly inhibited cell proliferation at $80 \mu \mathrm{mol} / 1$ compared with the control $(\mathrm{P}<0.001$; Fig. 1B). In 143B and MG-63 lower doses of SSd (20, 40 and $60 \mu \mathrm{mol} / \mathrm{l})$ had no significant influence on proliferation following 24 or $48 \mathrm{~h}$ treatment compared with the control ( $\mathrm{P}>0.05$; Fig. 1B). Subsequent experiments were performed using $80 \mu \mathrm{mol} / 1 \mathrm{SSd}$ as cell treatment.

$S S d$ causes cell cycle arrest in $G_{0^{-}} G_{l}$ phase. Flow cytometric analyses of the cell cycle were used to evaluate the underlying mechanisms of proliferation suppression by SSd. It was observed that $80 \mu \mathrm{mol} / 1 \mathrm{SSd}$ significantly increased the percentage of 143B and MG-63 cells in $\mathrm{G}_{0}-\mathrm{G}_{1}$ phase by 18 and $23 \%$, respectively $(\mathrm{P}<0.001 ;$ Fig. 2$)$.

SSd induces apoptosis. A significant increase in apoptosis was observed compared with the control group following the treatment of 143B and MG-63 with $80 \mu \mathrm{mol} / 1 \mathrm{SSd}$ for $48 \mathrm{~h}$ $(\mathrm{P}<0.001$; Fig. 3), indicating that SSd may serve a vital role in the apoptosis of OS cells.
Effects of SSd on cell cycle and apoptosis are associated with p53 accumulation. While cell cycle arrest and apoptosis are known to be associated with the activation of the p53 signaling pathway, SSd has been demonstrated to lead to p53 accumulation in ovarian cancer cell lines, Hey and SKOV3 (9). A potential association of SSd and p53 was evaluated. p53 expression was assessed in 143B and MG-63 treated with $80 \mu \mathrm{mol} / 1 \mathrm{SSd}$. Western blot analysis revealed that $\mathrm{SSd}$ promoted p53 accumulation in OS cells. Furthermore, it was demonstrated that $\mathrm{SSd}$ treatment was associated with the upregulation of p53 downstream targets, including p21, p27, Bax, cleaved caspase-3 and the downregulation of cyclinD1 (Fig. 4A). RT-qPCR was performed to evaluate the mRNA expression of p53 signaling pathway targets. In 143B and MG-63, SSd treatment $(80 \mu \mathrm{mol} / \mathrm{l})$ significantly decreased mRNA levels of cyclinD1 and significantly increased mRNA levels of p21, p27, Bax and cleaved caspase-3 ( $\mathrm{P}<0.001$; Fig. 4B). Thus, SSd increased the expression of $\mathrm{p} 53$ in protein and mRNA levels in 143B and MG-63.

\section{Discussion}

OS is the leading cause of death among primary bone malignancy, which originates from bone mesenchymal cells (22). It occurs mainly in adolescents and children ( $90 \%$ of patients with OS are $<20$ years of age) $(22,23)$. The OS survival rate in the US was $\sim 20 \%$, and the five-year OS survival rates for children and adolescents in Europe were similar $(22,23)$. Long-term survival rates of patients who underwent surgical resection of OS were $\sim 20 \%$ worldwide (23). In recent years, cisplatin-based combination chemotherapy has improved the prognosis of patients with OS and the 5-year overall survival rate for non-metastasis accounts for $60-70 \%$ (24). Resistance to chemotherapy describes an important factor influencing the treatment outcomes in OS $(23,25)$.

Over the past decades, traditional Chinese medicines, based on herbs and botanicals, have been applied in the treatment of osteoarthrosis, diabetes disease and malaria, and clinical evaluation deemed the formulations as extremely safe, efficient and to exhibit lower toxicity (25). For example, artemisinin was used in the treatment of malaria (24) and Chinese medicines, such as rosmarinic, were used as diabetic retinopathy therapies (26). SSd regulates T lymphocyte function, prevents the progression proteinuria, modulates macrophage function and enhances nonspecific resistance against aeruginosa infection $(7,27,28)$. The compound further possesses anti-inflammatory effects and causes cell death in human HCC cell lines (29). Other studies reported that SSd sensitizes tumor cells to cisplatin via ROS-mediated apoptosis and the combination of SSd with cisplatin may describe an effective therapeutic strategy (29). Wang et al (30) suggested that SSd potentiates effects of radiation in SMMC-7721 hepatocytes; thus, it may be a promising radiosensitizer further affecting the cell cycle (30). However, functions and underlying mechanisms of SSd in OS remain to be investigated.

The current study focused on antitumor activities of SSd. The results suggested that $143 \mathrm{~B}$ and MG-63 incubated with $80 \mu \mathrm{mol} / 1 \mathrm{SSd}$ exhibited significantly reduced cell viability when compared with the control group. In addition, DNA 
A
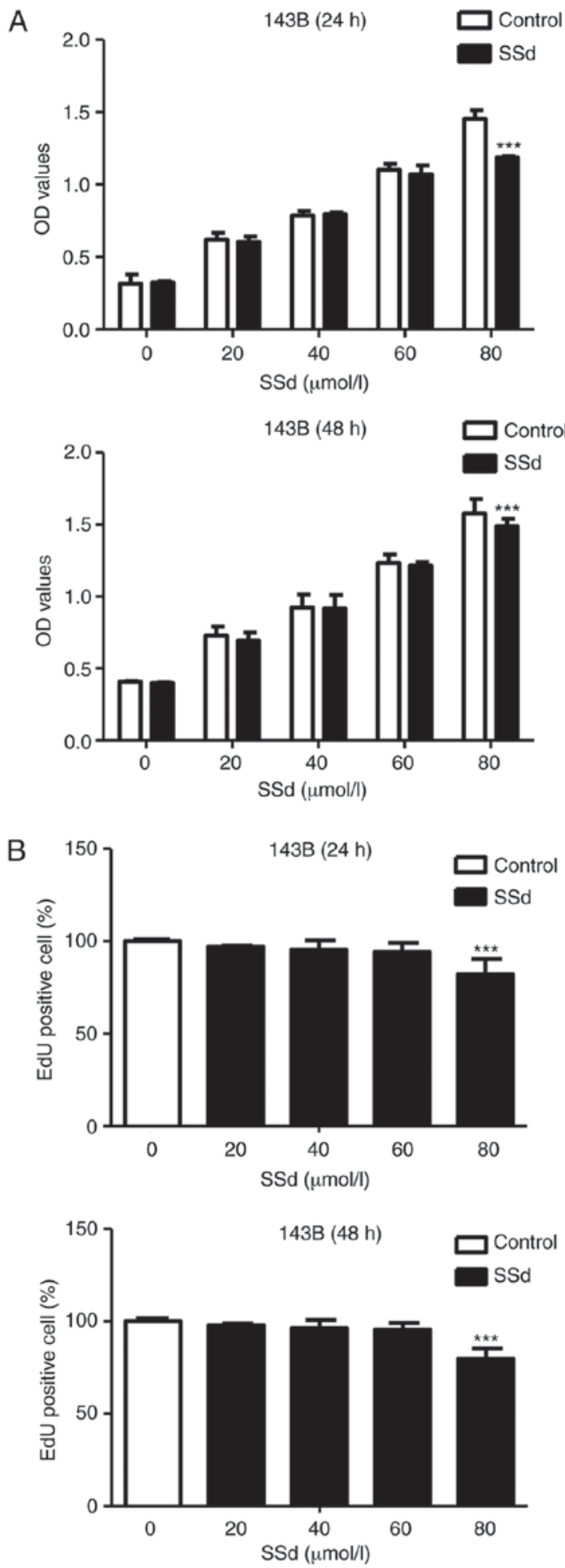
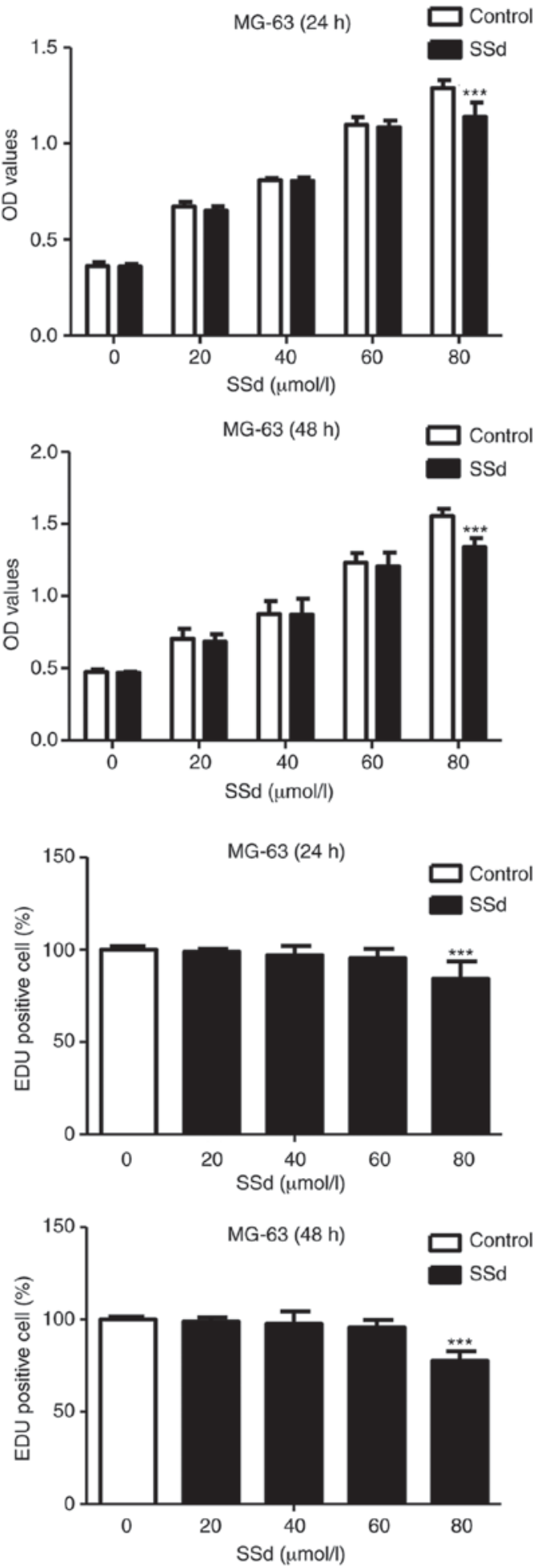

Figure 1. Inhibitory proliferation effects of SSd in 143B and MG-63. (A) MTS analysis was performed to detect proliferation at various SSd concentrations $(20,40,60$ and $80 \mu \mathrm{mol} / \mathrm{l})$ following 24 and $48 \mathrm{~h}$ treatment at a wavelength of $450 \mathrm{~nm}$. (B) EdU assays were performed to measure $143 \mathrm{~B}$ and MG-63 viability at various SSd concentrations (20, 40,60 and $80 \mu \mathrm{mol} / \mathrm{l})$ following 24 and $48 \mathrm{~h}$ treatment. ${ }^{* * *} \mathrm{P}<0.001$ vs. control. SSd, saikosaponin D; OD, optical density.

synthesis in 143B and MG-63 cells was significantly suppressed at the $\mathrm{G}_{1}$ phase after incubation with high doses of SSd. It has been reported that SSd is associated with various anticancer functions, influencing cell proliferation, apoptosis and migration and invasion of various cancer types $(7,27)$. The current study focused on antitumor activities od SSd in OS and it was demonstrated that $S$ Sd induced $\mathrm{G}_{0} / \mathrm{G}_{1}$ phase arrest and caused cells apoptosis, consistent with previous reports $(30,31)$. p53 serves a critical role in regulation of cell cycle checkpoints, DNA damage and the prevention of normal cell developing malignant phenotypes (32,33). Additionally, the tumor suppressor protein p53 is a vital component in the apoptotic pathway, and Bcl-2 and Bax are important transcriptional targets of p53. Bax, an anti-apoptotic protein, is essential for apoptosis and p53 can initiate cell death through activating target genes via the upregulation of Bax expression (31). Bcl-2 

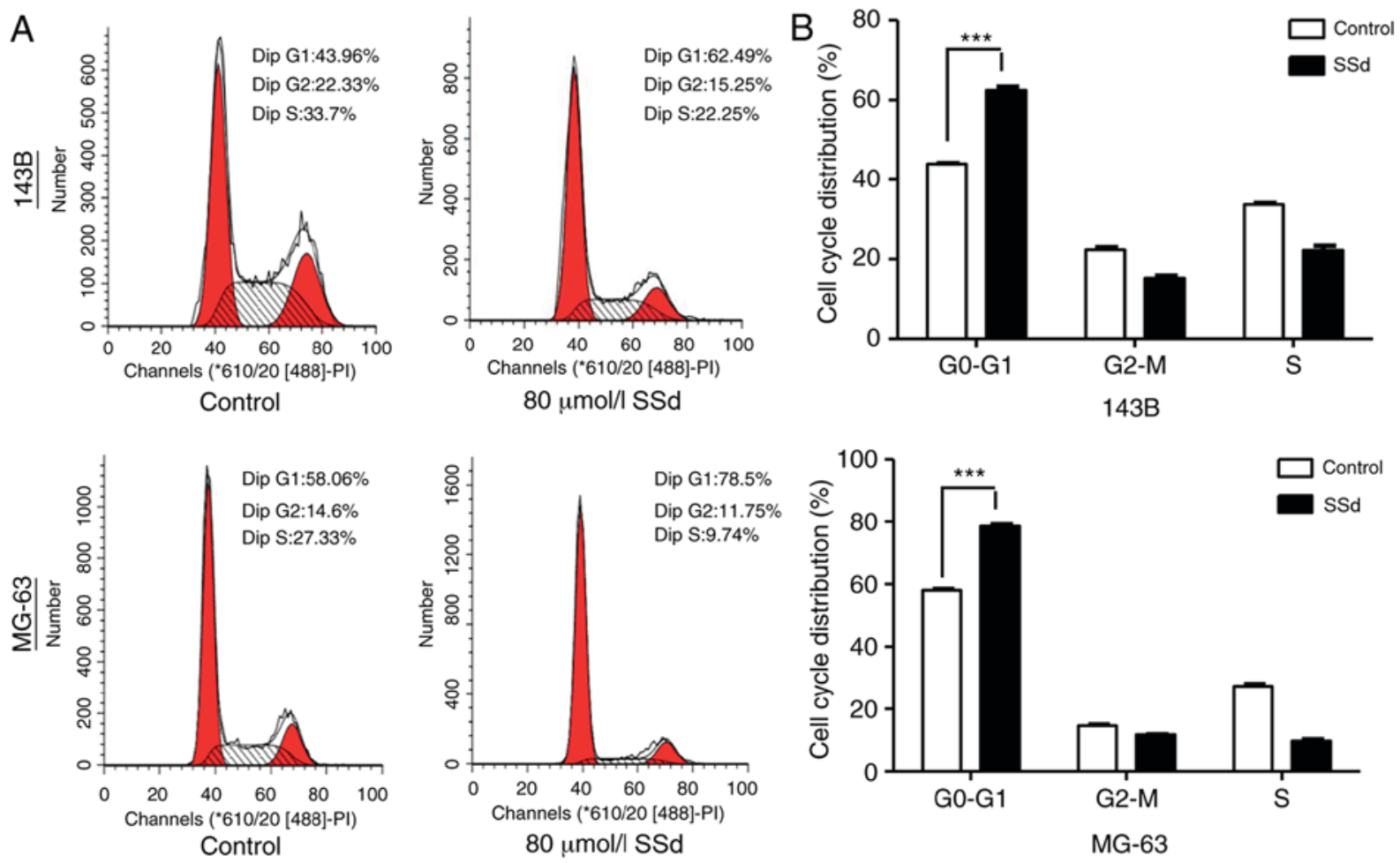

Figure 2. SSd induces cell cycle arrest at $\mathrm{G}_{0}-\mathrm{G}_{1}$ phase. Flow cytometry analysis of the cell cycle of $143 \mathrm{~B}$ and MG- 63 treated with $80 \mu \mathrm{mol} / 1 \mathrm{SSq}$ for $48 \mathrm{~h}$. (A) Representative images of cell cycle distribution in 143B and MG-63 and (B) statistical analysis of the data. ${ }^{* * *} \mathrm{P}<0.001$ vs. control. SSd, saikosaponin D.
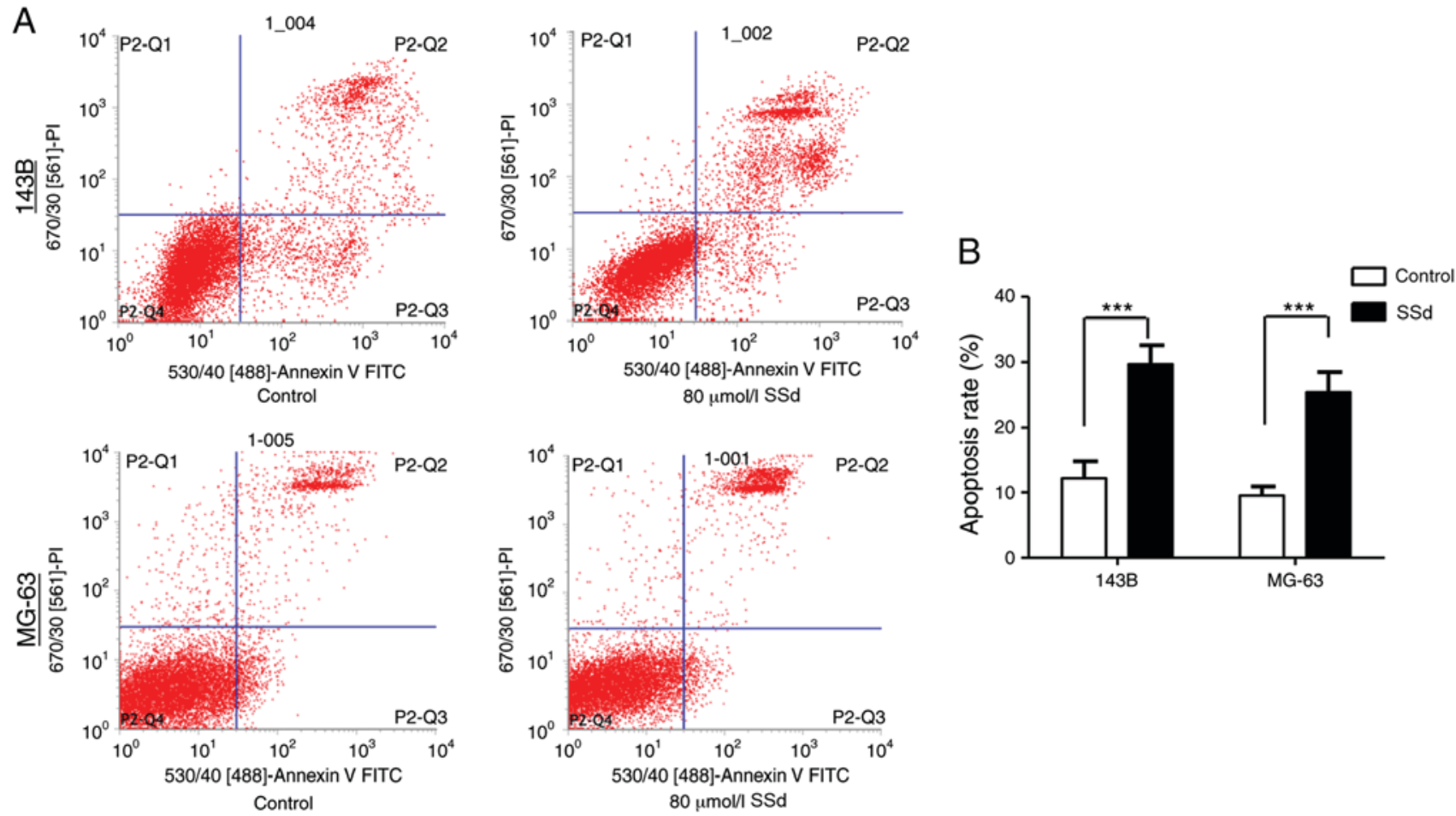

Figure 3. SSd induces apoptosis in osteosarcoma. Flow cytometry-based detection of apoptosis induced by SSd ( $80 \mu \mathrm{mol} / \mathrm{l})$ treatment for $48 \mathrm{~h}$. (A) Representative images of apoptosis analysis using Annexin V-FITC and PI double staining and (B) quantitative analysis of flow cytometry data. ${ }^{* * * *} \mathrm{P}<0.001$. SSd, saikosaponin D; FITC, fluorescein isothiocyanate; PI, propidium iodide.

family proteins serve central roles in mitochondria-mediated apoptosis and are divided into three groups: BH3-only proteins, BAX/bcl-2 homologous antagonist/killer (BAK) proteins and pro-survival proteins (34). Activated BAX/BAK forms homo-oligomers that permeabilize mitochondria and allow the release of pro-apoptotic factors, such as cytochrome c, which promote the activation of caspases $(35,36)$. In the present study, SSd significantly increased transcription of p53 and its 
A

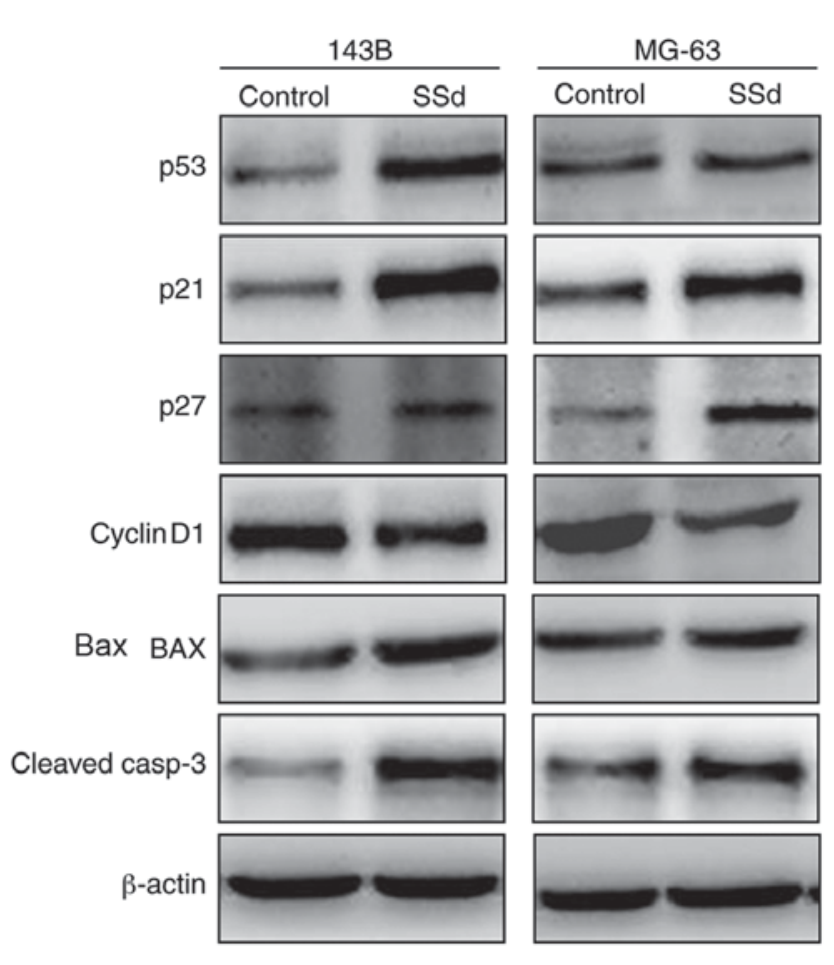

B
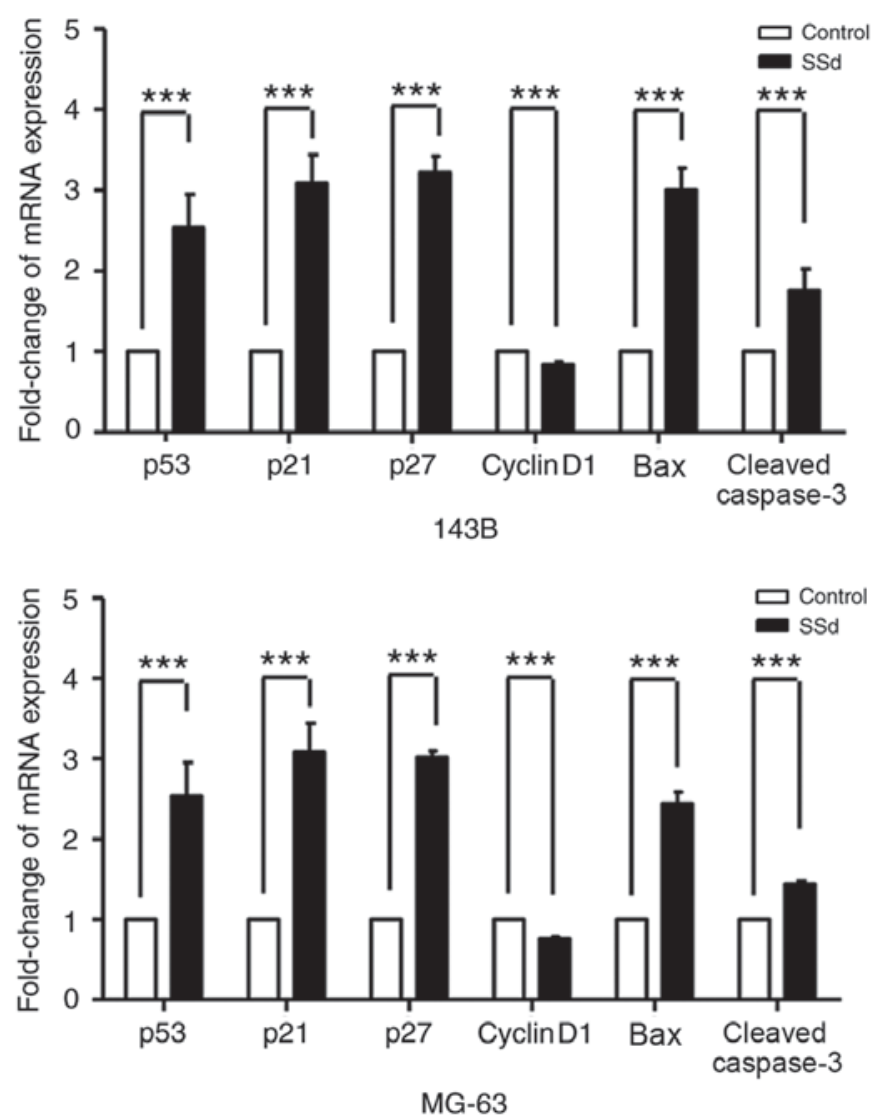

Figure 4. SSd affects p53 expression and downstream targets in 143B and MG-63. Cells were treated with SSd (80 $\mu$ mol/1) for $48 \mathrm{~h}$ and (A) protein and (B) mRNA levels of p53, p21, p27, cyclinD1, Bax and cleaved-caspase-3 were determined by western blot and reverse transcription-quantitative polymerase chain reaction, respectively. ${ }^{* * *} \mathrm{P}<0.001$. SSd, saikosaponin $\mathrm{D} ; \mathrm{p}$, tumor protein; Bax, B-cell lymphoma-2-like protein 4.

downstream targets, including p21, p27 and Bax, and decreased cyclinD1 expression. CyclinD1 has been demonstrated to activate the G1-S transition of the cell cycle $(35,36)$. Gumz et al $(37)$ reported that cyclinD1 was up-regulated in clear cell RCC and that an antagonist of the Wnt signaling pathway, sFRP1, inhibited cyclinD1 expression. The results of the current study demonstrated that SSD had a similar effect on cyclinD1.

In conclusion, the current study describes the role of SSd in inhibiting cell growth and inducing apoptosis through activation of the p53 signaling pathway in OS. SSd may serve as a potential tumor inhibitor in OS. However, large-scale in vivo experiments may be needed to validate its mechanism.

\section{Acknowledgements}

Not applicable.

\section{Funding}

No funding was received.

\section{Availability of data and materials}

The datasets used and/or analyzed during the current study are available from the corresponding author on reasonable request.

\section{Authors' contributions}

LZ and JL conducted the nucleic acid extraction, MTS and EdU assays and western blot analyses, and wrote the manuscript. Z-BS, CS and Z-HY collected the data and performed the cell culture, flow cytometry and the reverse transcription-quantitative polymerase chain reaction analysis. $\mathrm{XG}$ designed the study. The final version of the manuscript has been read and approved by all authors, and each author believes that the manuscript represents honest work.

\section{Ethics approval and consent to participate}

Not applicable.

\section{Patient consent for publication}

Not applicable.

\section{Competing interests}

The authors declare that they have no competing interest.

\section{References}

1. Widhe B and Widhe T: Initial symptoms and clinical features in osteosarcoma and Ewing sarcoma. J Bone Joint Surg Am 82: 667-674, 2000 
2. Lin JS, Chen R, Yan W and Chen DD: Enhancing soft-tissue reattachment with artificial mesh in joint endoprosthetic reconstruction for bone tumors. Zhonghua Zhong Liu Za Zhi 39: 540-544, 2017 (In Chinese).

3. Lu CN, Yuan ZG, Zhang XL, Yan R, Zhao YQ, Liao M and Chen JX: Saikosaponin a and its epimer saikosaponin d exhibit anti-inflammatory activity by suppressing activation of NF- $\mathrm{NB}$ signaling pathway. Int Immunopharmacol 14: 121-126, 2012.

4. Wong VK, Zhang MM, Zhou H, Lam KY, Chan PL, Law CK, Yue PY and Liu L: Saikosaponin-d enhances the anticancer potency of TNF- $\alpha$ via overcoming its undesirable response of activating NF-Kappa B signalling in cancer cells. Evid Based Complement Alternat Med 2013: 745295, 2013.

5. Tundis R, Bonesi M, Deguin B, Loizzo MR, Menichini F, Conforti F, Tillequin F and Menichini F: Cytotoxic activity and inhibitory effect on nitric oxide production of triterpene saponins from the roots of Physospermum verticillatum (Waldst \& Kit) (Apiaceae). Bioorg Med Chem 17: 4542-4547, 2009.

6. Hsu YL, Kuo PL and Lin CC: The proliferative inhibition and apoptotic mechanism of Saikosaponin D in human non-small cell lung cancer A549 cells. Life Sci 75: 1231-1242, 2004.

7. Hsu YL, Kuo PL, Chiang LC and Lin CC: Involvement of p53, nuclear factor kappaB and Fas/Fas ligand in induction of apoptosis and cell cycle arrest by saikosaponin $\mathrm{d}$ in human hepatoma cell lines. Cancer Lett 213: 213-221, 2004.

8. Motoo Y and Sawabu N: Antitumor effects of saikosaponins, baicalin and baicalein on human hepatoma cell lines. Cancer Lett 86: 91-95, 1994

9. Tsuyoshi H, Wong VKW, Han Y, Orisaka M, Yoshida Y and Tsang BK: Saikosaponin-d, a calcium mobilizing agent, sensitizes chemoresistant ovarian cancer cells to cisplatin-induced apoptosis by facilitating mitochondrial fission and G2/M arrest. Oncotarget 8: 99825-99840, 2017.

10. Zhuang Y, Gan Y, Tang T: Saikosaponin-d promotes apoptosis of osteosarcoma cells. International Journal of Orthopaedics 38 115-120, 2017.

11. Zhang P, Wang X and Guo X: Saikosaponin-D inhibits migration and invasion of osteosarcoma cell line MG-63 by reversing EMT. J Modern Oncol 25: 2561-2564, 2017.

12. Yang C, Fan W, Ou X and Yuan B: Inhibitory effect of Saikosaponin D on proliferation of osteosarcoma 143B cells. Chin Pharm 1: 9-13, 2018.

13. Wong VK, Li T, Law BY, Ma ED, Yip NC, Michelangeli F, Law CK, Zhang MM, Lam KY, Chan PL and Liu L: Saikosaponin-d, a novel SERCA inhibitor, induces autophagic cell death in apoptosis-defective cells. Cell Death Dis 4: e720, 2013.

14. Fan Y, Zhan Q, Xu H, Li L, Li C, Xiao Q, Xiang S, Hui T, Xiang T and Ren G: Epigenetic identification of ZNF545 as a functional tumor suppressor in multiple myeloma via activation of p53 signaling pathway. Biochem Biophys Res Commun 474 660-666, 2016

15. Xiong J, Liu Y, Jiang L, Zeng Y and Tang W: High expression of long non-coding RNA lncRNA-ATB is correlated with metastases and promotes cell migration and invasion in renal cel carcinoma. Jpn J Clin Oncol 46: 378-384, 2016.

16. Wu X, Zhang P, Zhu H, Li S, Chen X and Shi L: Long noncoding RNA FEZF1-AS1 indicates a poor prognosis of gastric cancer and promotes tumorigenesis via activation of Wnt signaling pathway. Biomed Pharmacother 96: 1103-1108, 2017.

17. Zhao L, Zhang H, Bao J, Liu J and Ji Z: Saikosaponin-d protects renal tubular epithelial cell against high glucose induced injury through modulation of SIRT3. Int J Clin Exp Med 8: 6472-6481, 2015.

18. Cao W, Peng T and Zhou Y: Long noncoding RNA activated by transforming growth factor- $\beta$ promotes cancer development and is a prognostic marker in cervical cancer. J Cancer Res Ther 13 801-806, 2017.
19. Gou X, Zhao X and Wang Z: Long noncoding RNA PVT1 promotes hepatocellular carcinoma progression through regulating miR-214. Cancer Biomark 20: 511-519, 2017.

20. Livak KJ and Schmittgen TD: Analysis of relative gene expression data using real-time quantitative PCR and the 2(-Delta Delta C(T)) method. Methods 25: 402-408, 2001.

21. Xu Y, Hu J, Zhang C and Liu Y: MicroRNA-320 targets mitogen-activated protein kinase 1 to inhibit cell proliferation and invasion in epithelial ovarian cancer. Mol Med Rep 16 8530-8536, 2017.

22. Mirabello L, Troisi RJ and Savage SA: Osteosarcoma incidence and survival rates from 1973 to 2004: Data from the surveillance, epidemiology, and end results program. Cancer 115: 1531-1543, 2009.

23. He SX, Luo JY, Zhao G, Xu JL, Wang YL, Fu H and Dong L: Effect of saikosaponins-d on cyclooxygenase-2 expression of human hepatocellular carcinoma cell line SMMC-7721. Zhonghua Gan Zang Bing Za Zhi 14: 712-714, 2016 (In Chinese).

24. Tu Y: The discovery of artemisinin (qinghaosu) and gifts from Chinese medicine. Nat Med 17: 1217-1220, 2011.

25. Girdhani S, Bhosle SM, Thulsidas SA, Kumar A and Mishra KP. Potential of radiosensitizing agents in cancer chemo-radiotherapy. J Cancer Res Ther 1: 129-131, 2005

26. Song W and Zhu YW: Chinese medicines in diabetic retinopathy therapies. Chin J Integr Med 28, 2018

27. Wang Q, Zheng XL, Yang L, Shi F, Gao LB, Zhong YJ, Sun H, He F, Lin Y and Wang X: Reactive oxygen species-mediated apoptosis contributes to chemosensitization effect of saikosaponins on cisplatin-induced cytotoxicity in cancer cells. J Exp Clin Cancer Res 29: 159, 2010.

28. Qian L, Murakami T, Kimura Y, Takahashi M and Okita K Saikosaponin A-induced cell death of a human hepatoma cell line (HuH-7): The significance of the 'sub-G1 peak' in a DNA histogram. Pathol Int 45: 207-214, 1995.

29. Cheng JT and Tsai CL: Anti-inflammatory effect of saikogenin A. Biochem Pharmacol 35: 2483-2487, 1986

30. Wang BF, Dai ZJ, Wang XJ, Bai MH, Lin S, Ma HB, Wang YL, Song LQ, Ma XL, Zan Y, et al: Saikosaponin-d increases the radiosensitivity of smmc-7721 hepatocellular carcinoma cells by adjusting the $\mathrm{g} 0 / \mathrm{g} 1$ and $\mathrm{g} 2 / \mathrm{m}$ checkpoints of the cell cycle. BMC Complement Altern Med 13: 263, 2013.

31. Li M, Li Y, Sun L, Song JL and Lv C: High mobility group box 1 promotes apoptosis of astrocytes after oxygen glucose deprivation/reoxygenation by regulating the expression of $\mathrm{Bcl}-2$ and Bax. Beijing Da Xue Xue Bao Yi Xue Ban 50: 785-791, 2018 (In Chinese)

32. Friedman MA and Carter SK: The therapy of osteogenic sarcoma: Current status and thoughts for the future. J Surg Oncol 4: 482-510, 1972

33. Kastan MB, Onyekwere O, Sidransky D, Vogelstein B and Craig RW: Participation of $\mathrm{p} 53$ protein in the cellular response to DNA damage. Cancer Res 51: 6304-6311, 1991.

34. Edlich F: BCL-2 proteins and apoptosis: Recent insights and unknowns. Biochem Biophys Res Commun 500: 26-34, 2018.

35. Kanthan R, Radhi JM and Kanthan SC: Gallbladder carcinomas: An immunoprognostic evaluation of P53, Bcl-2, CEA and alpha-fetoprotein. Can J Gastroenterol 14: 181-184, 2000.

36. Wang YW, Zhang K, Zhao S, Lv Y, Zhu J, Liu H, Feng J, Liang W, Ma R and Wang J: HPV status and its correlation with $\mathrm{BCL} 2, \mathrm{p} 21, \mathrm{p} 53, \mathrm{Rb}$, and survivin expression in breast cancer in a Chinese population. Biomed Res Int 2017: 6315392, 2017.

37. Gumz ML, Zou H, Kreinest PA, Childs AC, Belmonte LS, LeGrand SN, Wu KJ, Luxon BA, Sinha M, Parker AS, et al: Secreted frizzled-related protein 1 loss contributes to tumor phenotype of clear cell renal cell carcinoma. Clin Cancer Res 13: 4740-4748, 2007. 